been seasonally uncoupled from normal pituitary-derived production.

Although we are encouraged by the growth results observed with Pacific salmon, it remains to be seen whether pOnMTGH1 and other piscine gene constructs will generally function better than non-homologous ones in other cold- and warm-water fish species where growth hormone is limiting with regard to growth performance.

Robert H. Deviln ${ }^{\star}$, Timothy Y. Yesaki, Carlo A. Blagl, Edward M. Donaldson Fisheries and Oceans Canada,

4160 Marine Drive,

West Vancouver,

British Columbia V7V 1N6, Canada

Penny Swanson

Northwest Fisheries Science Center,

National Marine Fisheries Service,

Seattle, Washington 98112-2097, USA

Woon-Khlong Chan

Institute of Molecular and Cell Biology and Department of Zoology,

National University of Singapore,

Singapore 0511

"To whom correspondence should be addressed.

\section{Quasar variability}

SIR-Hawkins ${ }^{1}$ monitored the observed variability of quasars with redshifts between 1 and 3 , and finds that the timescale of variability decreases with redshift, which he argues is evidence in favour of the quasar light being microlensed by substellar objects along the line of sight. I show here that in fact the timescale of lensing should increase with redshift, because the average redshift of the lensing objects increases with the redshift of the quasar, resulting in a longer variability timescale as the time taken for the object to cross the line of sight is increased by time dilation.

I have calculated the autocorrelation function of the quasar magnitude variations produced by gravitational lensing by a population of intervening, randomly distributed, compact objects, under the assumption that the amplifications $A$ due to lensing by different objects combine multiplicatively. This is exact in the limit of very small lensing probability, and has been shown to be a good approximation up to modest lensing optical depths ${ }^{2}$. It is expected to be a reasonable approximation in the present case. The magnitude change is $\mu \equiv-2.5 \log _{10} A$, so that the contributions of different objects to $\mu$ add. For the case that the quasar is a point source at redshift $z_{S}$, lensing by a point mass $M_{L}$ at redshift $z_{L}$ and distance $l$ from the line of sight amplifies the brightness by a factor $A(u)=\left(u^{2}+2\right) / u \sqrt{u^{2}+4}$, where $u=l / R_{E}$, and $R_{E}\left(M_{L}, z_{L}, z_{S}\right)$ is the Einstein ring radius ${ }^{3}$. The amplification varies with time due to motions of the observer, source and lens; here I consider only the latter, with lenses moving with a
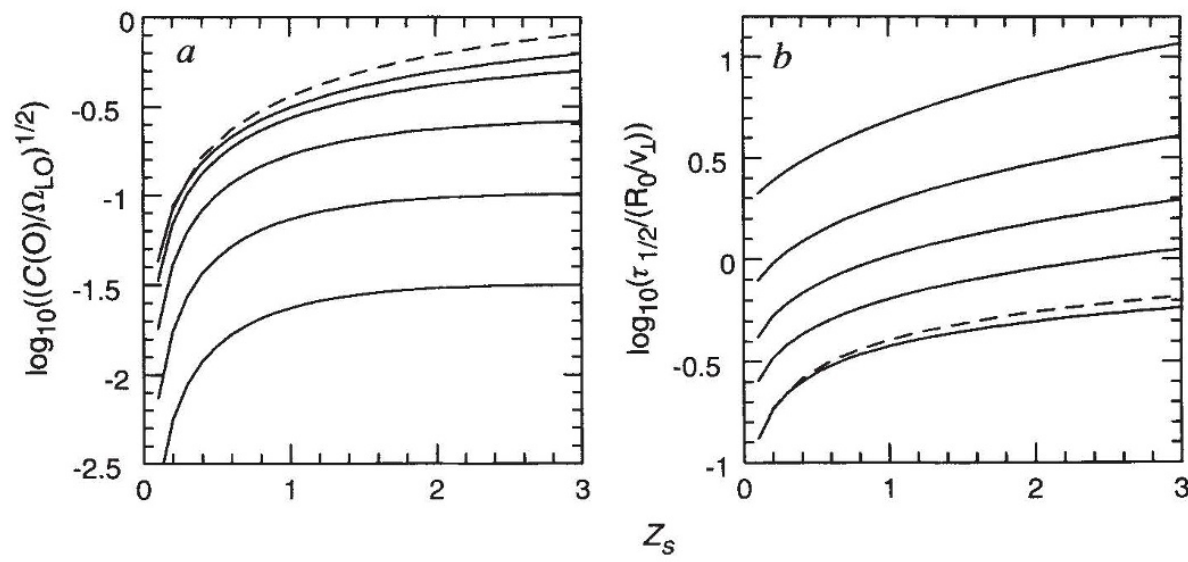

$a$, Root-mean-square magnitude variation, $c^{1 / 2}(0) ; b$, variability timescale, $\tau_{1 / 2}$, as functions of $z_{s}$, for different source radii $R_{s}$. Solid curves are for $\Omega_{0}=1$ and $R_{s} / R_{0}=0,0.3,1,3,10$ (decreasing vertically for $C(0)$ and increasing vertically for $\tau_{1 / 2}$ ). Dashed curves are for $\Omega_{0}=0.1$ and $R_{s} / R_{O}=0$.

transverse velocity $v_{\perp}$. The autocorrelation function at time lag $\tau$ for a single lens is $\int_{-\infty}^{\infty} \mu(t+\tau) \mu(t) \mathrm{d} t$. This is then integrated over $l$ and $z_{L}$, assuming a constant comoving number density of lenses, to give the net autocorrelation function $C(\tau) \equiv$ $\langle(\mu(t+\tau)-\langle\mu\rangle)(\mu(t)-\langle\mu\rangle)\rangle$. The characteristic timescale of magnitude variations due to lenses at redshift $z_{L}$ is just $\left(1+z_{L}\right) R_{E} / v_{\perp}$, where the factor $\left(1+z_{L}\right)$ results from time dilation.

The autocorrelation function $C(\tau)$, which is symmetrically peaked around $\tau=0$, can be simply characterized by an amplitude and a timescale. The amplitude $C(0)=\left\langle(\mu-\langle\mu\rangle)^{2}\right\rangle=0.806 p_{L}$ gives the mean square magnitude variation. Here, $p_{L}\left(z_{S}, \Omega_{L 0}\right)$ is the probability for a strong lensing event $\left(l<R_{E}\right)$, also called the optical depth for lensing ${ }^{3}$, and is proportional to the fraction of the critical density in lensing objects, $\Omega_{L 0}=8 \pi G n_{L 0} M_{L} /\left(3 H_{0}^{2}\right)$, but independent of the lens mass $M_{L}$. $\left(n_{L 0}\right.$ is the present number density of lenses, and $H_{0}$ is the present Hubble constant, taken to be $50 \mathrm{~km} \mathrm{~s}^{-1} \mathrm{Mpc}^{-1}$ ). The timescale, for example the half-width at half maximum $\tau_{1 / 2}$, is independent of $\Omega_{L 0}$, but scales as $\tau_{1 / 2} \propto R_{0} / v_{\perp}$ for given $z_{S}$. Here, $R_{0} \equiv\left(4 G M_{L} / c H_{0}\right)^{1 / 2}$ is the characteristic value of $R_{E}$, and the characteristic timescale $R_{0} / v_{\perp}=2.6 \operatorname{yr}\left(M_{L} / 10^{-3} M_{\odot}\right)^{1 / 2} /\left(v_{\perp} /\right.$ $\left.400 \mathrm{~km} \mathrm{~s}^{-1}\right)$. The figure shows the dependence of $C^{1 / 2}(0)$ and $\tau_{1 / 2}$ on $z_{S}$, for total density parameters $\Omega_{0}=0.1$ and $\Omega_{0}=1$.

I have also computed the effect of a finite source size, approximating the quasar as a uniform surface brightness disk of radius $R_{S}$, following $\mathrm{P}$. Schneider (personal communication). The radii of the optical continuum emitting regions of quasars are uncertain, but are thought to be at least $10^{15} \mathrm{~cm}$ for the more luminous quasars. The results are shown in the figure for different values of $R_{S} / R_{0}=0.3\left(R_{S} /\right.$ $\left.10^{15} \mathrm{~cm}\right) /\left(M_{L} / 10^{-3} M_{\odot}\right)^{1 / 2}$. The effect of a finite source size is to decrease the amplitude of magnitude variations and increase their timescale. For $R_{S} / R_{0} \gg 1$, these scale as $C^{1 / 2}(0) \propto R_{S}^{-1}$ and $\tau_{1 / 2} \propto R_{S}$; only a fraction of the quasar light is significantly amplified, and the timescale is essentially that for a lensing object to cross the quasar disk.

Hawkins estimates a quantity similar to $\tau_{1 / 2}$ from his observational data, and finds $\tau_{1 / 2} \sim 1-5 \mathrm{yr}$, with the timescale decreasing by a factor $0.6-0.7$ from $z_{S} \approx 1.5$ to 2.5 . This is in conflict with the gravitational lensing calculations, which predict that $\tau_{1 / 2}$ should increase by a factor ranging from 1.2 for $R_{S} / R_{0} \ll 1$ to $1.4-1.6$ for $R_{S} / R_{0} \gg 1$, for $\Omega_{0}$ in the range $0.1-1$. The simplest intrinsic variability model predicts that $\tau_{1 / 2}$ should increase as $\left(1+z_{S}\right)$, that is, a factor 1.4 , due to time dilation at the source. In this sense, the lensing model (for point sources) is closer to the observed trend. Another test of the lensing hypothesis would be to compare predicted and observed autocorrelation amplitudes $C(0)$. It would also be useful to carry out numerical simulations of lensing, including the various observational selection effects, to check the accuracy of the assumptions I have made here.

\section{G. Lacey}

Physics Department,

University of Oxford,

Oxford OX1 3RH, UK

1. Hawkins, M. R. S. Nature 366, 242-245 (1993).

2. Pei, Y. C. Astrophys. J. 403, 7-19 (1993).

3. Paczynski, B. Astrophys. J. 304, L1-L5 (1986).

\section{Scientific Correspondence}

Scientific Correspondence is a relatively informal section of Nature in which matters of general scientific interest, not necessarily those arising from papers appearing in Nature, are published. Because there is space to print only a small proportion of the letters received, priority is usually given according to general interest and topicality, to contributions of fewer than 500 words, and to contributions using simple language. 